\title{
Estimation Method of Ionospheric TEC Distribution using Single Frequency Measurements of GPS Signals
}

\author{
Win Zaw Hein", Yoshitaka Goto", Yoshiya Kasahara ${ }^{\#}$ \\ "Division of Electrical Engineering and Computer Science, \\ Graduate School of Natural Science and Technology, \\ Kanazawa University \\ T920-1192, Kakuma-machi, Kanazawa, Ishikawa, Japan
}

\begin{abstract}
The satellite-to-ground communications are influenced by ionospheric plasma which varies depending on solar and geomagnetic activities as well as regions and local times. With the expansion of use of the space, continuous monitoring of the ionospheric plasma has become an important issue. In Global Positioning System (GPS), the ionospheric delay, which is proportional to ionospheric total electron content (TEC) along the propagation path, is the largest error in signal propagation. The TEC has been observed from dual frequency GPS signals because only the ionospheric delay has frequency dependences. Costs of multi-frequency receivers are, however, much higher than those of single frequency ones. In the present study, an estimation method of TEC distribution map from single frequency GPS measurements was developed. The developed method was evaluated by comparing its results with those from dual frequency measurements. The method makes it possible to expand ionospheric TEC observation networks easily.
\end{abstract}

Keywords-Global Positioning System; GPS; Signal processing and propagation; Ionospheric Delay; Total Electron Content (TEC)

\section{INTRODUCTION}

The Earth's ionosphere consists of electrons and ions called plasma [1]. They are generated by ionization of neutral particles of the atmosphere by ultraviolet rays from the Sun. Our daily lives are based on a variety of satellite-to-ground communications and they are greatly influenced by the ionospheric plasma, such as delays and scintillations. Monitoring of the ionospheric plasma is getting more important with the expansion of use of the space.

The ionosphere is the dominant source of space plasma around the Earth. Plasma density and constituent in the ionosphere show complex dependences on solar and geomagnetic activities. Responses of the ionospheric plasma to such activities has usually been investigated by direct observations from spacecraft and radar observations from the ground. In these observations, there are restrictions and limitation in observational periods, regions or time continuity. These restrictions are essential problems in a view point of the monitoring of the ionospheric plasma.

Continuous monitoring of the total electron content (TEC), which denotes integration of electron density, using observational networks of GNSS (global navigation satellite system) signals has recently become popular in many countries. The GNSS observation networks make it possible to reconstruct vertical TEC maps in wide regions in high spatial and temporal resolutions. The most widely distributed network service is provided by the International GNSS service (IGS). In Japan, more than 1,200 GNSS stations are installed all over the islands. This observational network is called GEONET and was originally built for land survey. This kind of observational network of GNSS signals are now an important tool to measure TEC and are used as an important information source of space weather forecast. Most of the networks are, however, built in mid latitude regions. It is important to expand them in lower latitudes regions where the effect of ionospheric plasma on radio waves for satellite-to-ground communications is the largest and the most complex.

In the GNSS observation stations, multi-frequency receivers are generally installed. They can accurately derive the ionospheric effects because only the ionospheric delay depends on the frequency among ranging errors. There are several reconstruction methods of TEC distribution map from each slant TEC measurement [2, 3, 4, 5, 6, 7, 8]. The global ionosphere maps (GIM) is provided by CODE using data from about 200 GNSS stations of the IGS and other stations [2]. The vertical TEC is modeled in a solar geomagnetic coordinate system using a spherical harmonics expansion. Global TEC maps are also provided by ESA/ESOC. The maps are also modelled by spherical harmonics in combination with a daily DCBs fitting using GPS and GLONASS data. Three shells are used in NASA/JPL ionospheric model from data obtained at 200 globally distributed stations [3, 4]. In this method, Kalman filter is used to smooth temporal variations. Most of the reconstruction methods are based on spherical harmonics.

The multi-frequency receivers used in the GNSS stations are, however, much more expensive than single frequency receivers which are widely distributed as consumer products. In the present study, an estimation method of ionospheric TEC map from single frequency measurements of GPS signals was developed. In the method, the TEC maps over a receiver are estimated under the condition that the receiver location is accurately known. The method can make it possible to build observation networks of ionospheric TEC at low cost. 


\section{IONOSPHERIC DELAY IN GPS SIGNAL AND TOTAL ELECTRON CONTENT, TEC}

One of the most fundamental GPS observables is C/A code pseudorange which is generally used for standard positioning $[9,10]$. The code pseudorange is measured from propagation time of the signal from $i^{\text {th }}$ satellite to the receiver and is represented by

$$
R^{i}=\rho^{i}+c\left(\delta t_{\mathrm{r}}-\delta t_{\mathrm{s}}^{i}\right)+\delta_{\text {ion }}^{i}+\delta_{\text {tro }}^{i}+\varepsilon,
$$

where $\rho^{i}$ is the geometric distance between satellite and receiver, $c$ is the velocity of light, $\delta t_{\mathrm{s}}^{i}$ and $\delta t_{\mathrm{r}}$ are transmitting and receiving time errors, respectively, $\delta_{\text {ion }}^{i}$ and $\delta_{\text {tro }}^{i}$ are the ionospheric and tropospheric effects along the propagation path of the signal. $\varepsilon$ denotes the other effect, such as multipath effect and receiver noise [11].

The ionospheric delay $\delta_{\text {ion }}^{i}$ is directly proportional to the total electron content (TEC) from the $i^{\text {th }}$ satellite to the receiver. This TEC is called slant TEC and represented by $I_{\text {slant }}^{i}$ as shown in the following formula,

$$
\delta_{\text {ion }}^{i}=\frac{e^{2}}{8 \pi^{2} m \varepsilon_{0} c f^{2}} I_{\text {slant }}^{i} \equiv \frac{I_{\text {slant }}^{i}}{\Lambda},
$$

where $e, m, \varepsilon_{0}$ and $f$ are charge and mass of the electron, permittivity of free space and the signal frequency, respectively. For convenience, the coefficient of $I_{\text {slant }}^{i}$ in the right term in equation (2) is defined as $1 / \Lambda$. When $\delta_{\text {ion }}^{i}$ is represented as meter and $I_{\text {slant }}^{i}$ as TECU $\left(1\right.$ TECU $=10^{16}$ electrons $\left./ \mathrm{m}^{2}\right), 1 / \Lambda$ is approximately equal to $40.3 / f^{2}$, where $f$ $=1575.42 \mathrm{MHz}$.

Since only the ionospheric effect depends on the signal frequency among the measurement errors in equation (1), $I_{\text {slant }}^{i}$ is generally calculated from distance measurements by dual frequency signals as follows;

$$
I_{\text {slant }}^{i}=\frac{\Lambda \cdot f_{2}{ }^{2}}{\left(f_{1}{ }^{2}-f_{2}{ }^{2}\right)}\left(R_{2}{ }^{i}-R_{1}{ }^{i}\right),
$$

where subscripts 1 and 2 corresponds to L1 and L2 signals. In practical, this value is compensated by differential code biases of the satellites and the receiver.

In the present paper, a new method to estimate $I_{\text {slant }}^{i}$ from single frequency measurement of GPS signals is proposed by adopting a spatial model for vertical TEC distribution in the ionosphere. Estimation accuracy of $I_{\text {slant }}^{i}$ is discussed by comparing with that from dual frequency observations as represented by equation (3).

\section{IONOSPHERIC TEC FROM SINGLE FREQUENCY MEASUREMENT}

Precise satellite orbit, velocity and clock error can be calculated from precise ephemeris, which is provided by the international GNSS service (IGS) [7]. Since the precise ephemeris is composed from location and clock error of each satellite whose time interval is 5 minutes, they are interpolated with 9th order Lagrange polynomial function. Then, $\rho^{i}$ and $\delta t_{\mathrm{s}}$ in equation (1) are obtained with an accuracy of few $\mathrm{cm}$ at any timing. In this calculation, relativistic effects are taken into account. As for $\delta_{\text {tro }}^{i}$, the Hopfield model whose accuracy is known to be less than $10 \mathrm{~cm}$ is adopted [12].

Under the condition that the receiver location is accurately known, the terms $\delta_{\text {ion }}^{i}$ and $\delta t_{\mathrm{r}}$ are undetermined in equation (1). While $\delta t_{\mathrm{r}}$ is common for all the satellite, $\delta_{\text {ion }}^{i}$ depends on the satellite locations. This is an essential feature to distinguish each effect. From equations (1) and (2), the slant TEC $I_{\text {slant }}^{i}$ is represented by

$$
I_{\text {slant }}^{i}=\Lambda\left(R^{i}-\rho^{i}+c \delta t_{\mathrm{s}}^{i}-\delta_{\text {tro }}^{i}\right)-\Lambda c \delta t_{\mathrm{r}}+\varepsilon^{\prime},
$$

The right side of the equation (4) can be separated into two parts, and defined $\kappa \equiv \Lambda\left(R^{i}-\rho^{i}+c \delta t_{\mathrm{s}}^{i}-\delta_{\text {tro }}^{i}\right)$ as a known part and $\alpha \equiv \Lambda c \delta t_{\mathrm{r}}$ as an unknown part.

Since the ionospheric TEC distribution is usually shown by vertical TEC map, the slant TEC is converted to vertical TEC by a slant factor. This conversion is important in the TEC map reconstruction from single frequency GPS data because the effects of slant TEC and the receiver clock error on propagation delays should be distinguished depending on the slant effects. In practical, the ionosphere can be assumed to be a thin layer and the ray path crosses the ionosphere at one point called the ionospheric pierce point (IPP) as shown in Fig. 1.

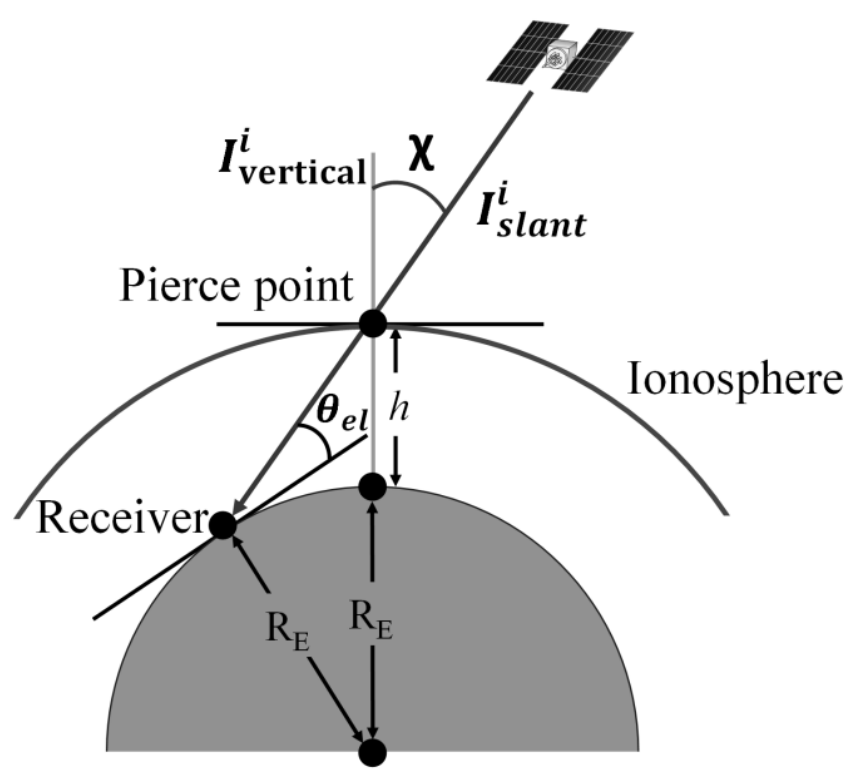

Fig. 1. Conversion model from slant TEC to vertical TEC in a thin layer assumption for the ionosphere

The conversion method from slant TEC $I_{\text {slant }}^{i}$ to vertical TEC $I_{\text {vertical }}^{i}$ can be used as follows;

$$
I_{\text {slant }}^{i}=I_{\text {vertical }}^{i} \cdot \frac{1}{\cos \chi i^{\prime}}
$$

where the angle $\chi$ between zenith direction and satellite direction from the IPP can be calculated as follows;

$$
\chi i=\sin ^{-1}\left(\frac{R_{E}}{R_{E}+h} \cos \theta_{\mathrm{el}}^{i}\right),
$$


where $R_{E}$ is Earth radius, $h$ is the altitude of IPP which is assumed to be $350 \mathrm{~km} \mathrm{[13]} \mathrm{and} \theta_{\mathrm{el}}^{i}$ is elevation angle of the satellite at the receiver location. by,

From Eq. (4), (5) and (6), vertical TEC can be represented

$$
I_{\text {vertical }}^{i}=(\kappa-\alpha) \cos \chi \equiv(\kappa-\alpha) F
$$

where $\cos \chi$ is defined as a slant factor $F$. This factor is used as a thin layer slant model for conversion of slant TEC to vertical TEC.

\section{SPATIAL DistribUtion MODEL OF IONOSPHERIC TEC}

There are some reconstruction methods of TEC distribution from dual frequency GPS data, as shown in Introduction. In the present study, TEC distribution is assumed to be represented by two dimensional (latitude-longitude) model with a first order function in each dimension because we deal with a small area within a few hundred kilometers squares. Vertical TEC distributions are represented as follows;

$$
I_{\text {vertical }}^{i}=I_{0}+\Delta I_{x} x+\Delta I_{y} y
$$

where $x$ and $y$ are normalized longitude (local time) and latitude. $I_{0}, \Delta I_{x}$, and $\Delta I_{y}$ are vertical TEC at the reference point $\left(135^{\circ} \mathrm{E}, 36^{\circ} \mathrm{N}\right)$, gradients of vertical TEC for $x$ and $y$ directions. $I_{0}, \Delta I_{x}$, and $\Delta I_{y}$ are parameters which should be estimated from measurements.

From Equations (7) and (8), the following relation is obtained;

$$
I_{0}+\Delta I_{x} x+\Delta I_{y} y=(\kappa-\alpha) F
$$

where $I_{0}, \Delta I_{x}, I_{y}$ and $\alpha$ are unknown parameters to be solved. At least four independent equations are required to solve the unknown parameters in Eq. (9), that is, the number of visible satellite from the receiver must be more than four. According to the GPS constellation, this condition is always satisfied. When $n$ numbers of satellites are visible from the receiver location, the following matrix is obtained,

$$
\left(\begin{array}{cccc}
1 & x_{0} & y_{0} & F_{1} \\
1 & x_{1} & y_{1} & F_{2} \\
\vdots & \vdots & \vdots & \vdots \\
1 & x_{n} & y_{n} & F_{n}
\end{array}\right)\left(\begin{array}{c}
I_{0} \\
\Delta I_{x} \\
\Delta I_{y} \\
\alpha
\end{array}\right)=\left(\begin{array}{c}
\kappa \cdot F_{1} \\
\kappa \cdot F_{2} \\
\vdots \\
\kappa \cdot F_{n}
\end{array}\right)
$$

In this equation, $\left(x_{i}, y_{i}\right)$ denotes location of the pierce point of $i^{\text {th }}$ visible satellites and $F_{i}$ is a slant factor of the $i^{\text {th }}$ satellite. As described in Section III, these values are accurately obtained from the precise ephemeris and location of the receiver. When equation (10) is represented as $\boldsymbol{X I}=\boldsymbol{m}$, unknown vector $\boldsymbol{I}$ can be estimated using linear least square method as follows;

$$
\boldsymbol{I}=\left(\boldsymbol{X}^{T} \boldsymbol{X}\right)^{-1} \boldsymbol{X}^{T} \boldsymbol{m} .
$$

The estimated parameters are used in the reconstruction of the vertical TEC map.

\section{EXAMPLE APPLICATION TO THE GEONET DATA}

The developed method was applied to pseudorange data obtained by the GEONET which is the GPS observation network in Japan. The pseudorange data are open to the public through a FTP site. Actually, although dual frequency data are available, only L1 frequency pseudorange data are used for examination of the proposed method.

An example result of TEC map is shown for data obtained at Uchinada station $\left(136^{\circ} \mathrm{E}, 36^{\circ} \mathrm{N}\right)$ in Japan on November 10 , 2013. From the precise ephemeris on the day, locations of the pierce points and elevation angle factors are calculated for all visible satellites. Figure 2. (a) shows the TEC map at local time of 11 hour in JST (Japan Standard Time) that is 2 hour UT (Universal Time) on Nov. 10 which is reconstructed from the estimated parameter vector $\boldsymbol{I}$ in Eq. (11). The map is shown from $125^{\circ} \mathrm{E}$ to $150^{\circ} \mathrm{E}$ in longitude and from $25^{\circ} \mathrm{N}$ to $47.5^{\circ} \mathrm{N}$ in latitude. The color bar at the right side of the figure shows the vertical TEC in TECU. The receiving station is shown as black circle in the figure. The pierce points are shown by blue triangle points. In this period, 9 satellites are visible from the receiving station. According to the satellite constellation, GPS satellites do not appear in north part of Japan.

The result shows the TEC above the receiver location is around 45 TECU and it is decreasing from 50-60 TECU to 3040 TECU as the location moves from lower to higher latitudes. As for local time variation of the TEC, the maximum value is generally found in the early afternoon. Since data acquisition time is 11 hour JST and the JST is defined at $135^{\circ} \mathrm{E}$, TEC in the east side of Japan should be larger than the west side. In the result, such longitudinal gradient is clearly found. The estimated values and their variations for latitude and longitude are typical.

The result is compared with the TEC map derived from dual frequency observations. The NASA Jet Propulsion Laboratory (JPL) provides global ionospheric map (GIM) with every 2 hours from data obtained at more than 400 GNSS stations all over the world. Figure 2. (b) shows the longitudinal and latitudinal TEC variation map around Japan from dual frequency measurement on the same period. The result shows almost the same tendency to the single frequency result in latitudinal and longitudinal variations while the TEC values of the single are a few TECU lower than those of the dual at same locations. 


\section{TEC Map [2013 Nov 10, 11:00 JST]}

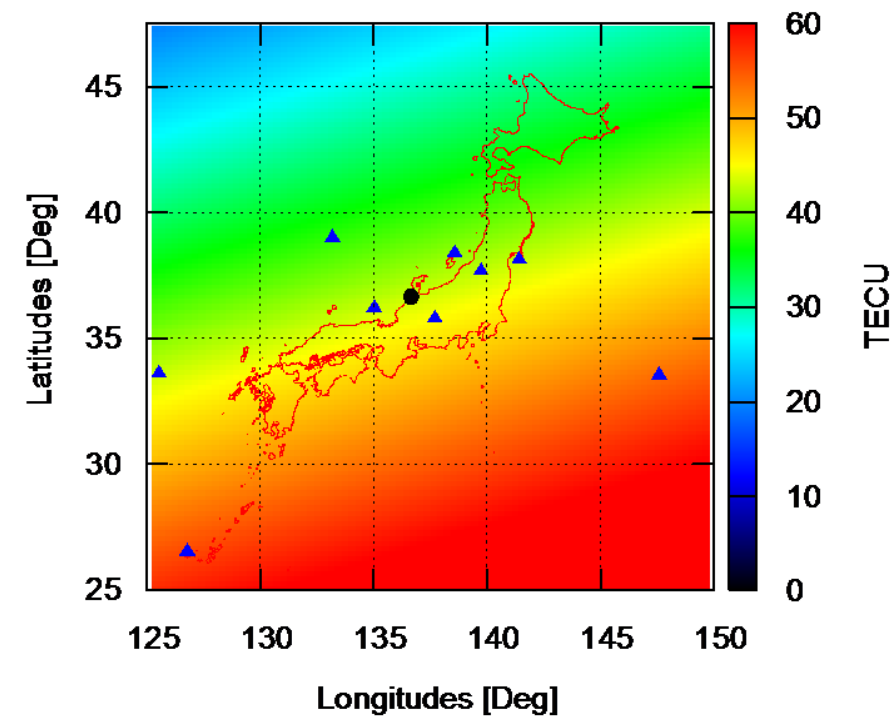

(a)

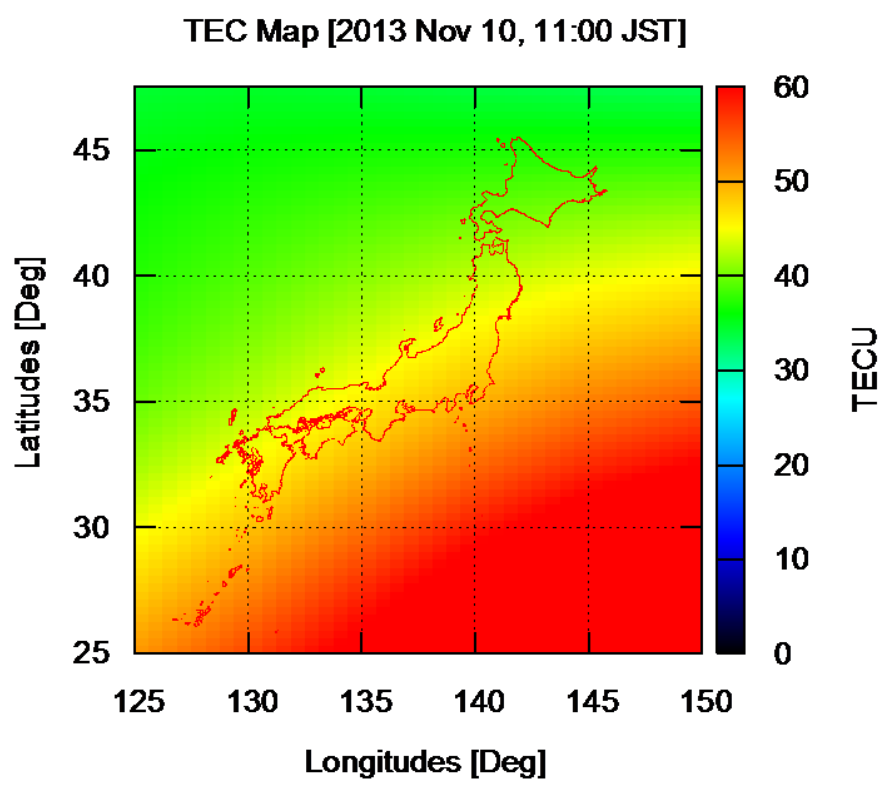

(b)

Fig. 2. (a) Latitudinal and longitudinal TEC map derived by single frequency measurement of proposed model. (b) Latitudinal and longitudinal TEC map derived by dual frequency measurements provided by IGS

\section{ANALYSIS OF ONE DAY VARIATION}

One-day data analysis can make it possible to check the statistical validity of the proposed method to various kinds of TEC variations. The proposed method was applied to the whole data obtained at the Uchinada station on November 10, 2013. The data are provided every 30 seconds and thus there are 2880 data sets. The parameters $I_{0}, \Delta I_{x}, \Delta I_{y}$ and $\alpha$ are independently estimated for each data. The estimated parameters are evaluated by comparing with vertical TECs converted from slant TECs that are measured by dual frequency signals at the same times.

Figure 3 shows results of estimated parameters of $I_{0}, \Delta I_{x}$, $\Delta I_{y}$, and receiver clock error $\alpha$ and its residual error. In this figure, the horizontal axis shows local time in JST from 9 hour on Nov. 10 to 9 hour on Nov. 11, that is, 0 to 24 hour in UT on Nov. 11. In figures 3 (a), the vertical TECs estimated at the reference point $I_{0}$ is represented by a red dotted line and the vertical TEC at each pierce point by dual frequency signal is represented by black line. From this result, both the vertical TECs show around 40 TECU in daytimes from 9 to 15 hour of JST. It is decreasing from 40 TECU to 10 TECU in the evening from 15 to 19 hour. It becomes less than 10 TECU and there is no remarkable variation at night. In the morning, it increases again from 10 TECU to 30 TECU. There seems a good correspondence between $I_{0}$ and dual frequency measurement.

The longitudinal and latitudinal gradients of TEC $\Delta I_{x}$, and $\Delta I_{y}$ are represented by green and by blue lines, respectively, in figure 3 (b). The gradients are defined by TEC variations for 15 degrees in TECU. The longitudinal gradient is plus values before 13 hour. After that, it takes minus values until the next morning. Because longitudinal gradient mainly signifies local time gradient due to the Earth's rotation, it should take plus values from morning region to the noon. In the afternoon, it should be minus. Thus, the estimated gradient is consistent with the typical local time variation. As a comparison of the results from figure 3 (a) and (b), the local time gradient of vertical TEC $I_{0}$ and longitudinal TEC $\Delta I_{x}$ are reasonably same each other.

As for latitudinal gradient $\Delta I_{y}$, it takes minus values except for after midnight. The value is largely fluctuated from around -5 to -40 TECU in the daytime. Since the latitudinal TEC gradient indicates TEC gradients from south to north, it is reasonable to take minus values in the daytime.

Figure 3 (c) shows the estimated receiver clock error $\alpha$ in meter by red dotted line and that derived from the dual frequency measurements by black solid line. Actually, there are two types of GPS receivers; one adjusts its receiver clock error successively and the other adjusts its clock error after it is accumulated to a certain extent, such as $1 \mathrm{~ms}$. From the absolute values of the receiver clock error in figure 3 (c), it is noted that the receiver of the Uchinada station is former type.

From comparison of the two curves in figure 3 (c), the receiver clock error is well estimated by the proposed method. This means that the assumption of TEC model with $1^{\text {st }}$ order gradients is appropriate during the day. There are small deviations at around local times of 13 hour, 15 hour and 21-26 hour. During these periods, there are also deviations on the estimated TEC in figure 3 (a). Since 1 TECU estimation error corresponds to $16 \mathrm{~cm}$ of receiver clock error, the receiver clock error should be estimated with much higher accuracy than its hourly variation to get ionospheric TEC.

Figure 3 (d) shows residual error of the least square method applied on equation (10). The error is shown in meter. From the result, the fitting error seems around $0.5 \mathrm{~m}$ which is equivalent to 3 TECU in average. There are a few peaks 
during day time in the figure. During these periods, the residual errors are more than 1 meter, which means that the estimated TEC may include errors greater than 6 TECU. The TEC values are, however, large during these periods, and during night time when small TEC values are obtained, the fitting error is also small.

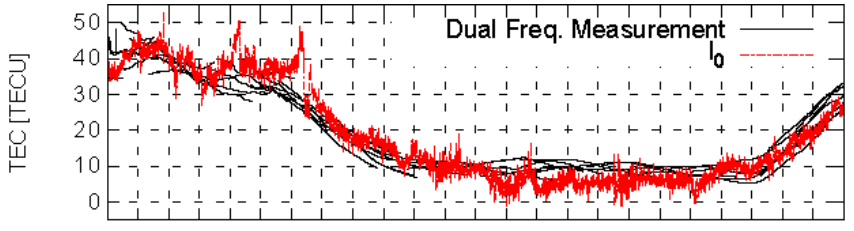

g 101112131415161718192021222324252627282930313233

(a)

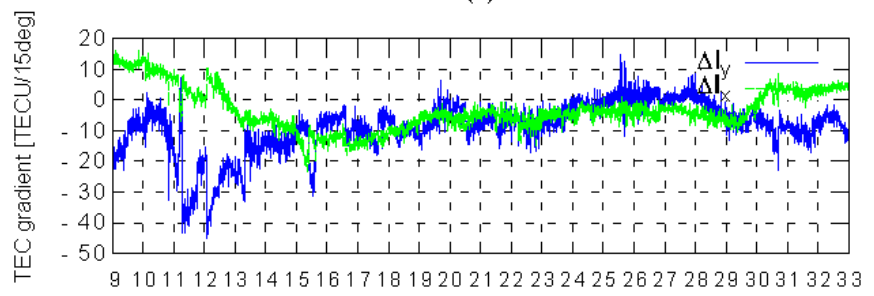

(b)

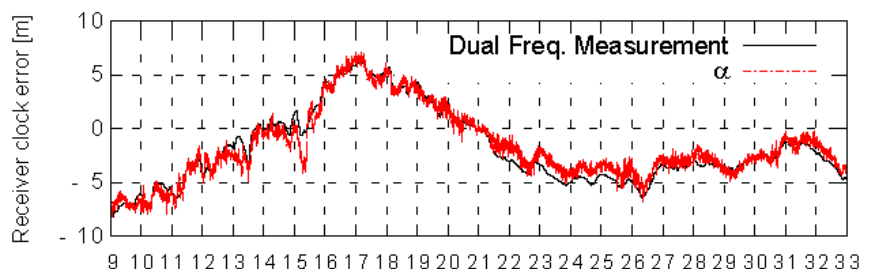

(c)

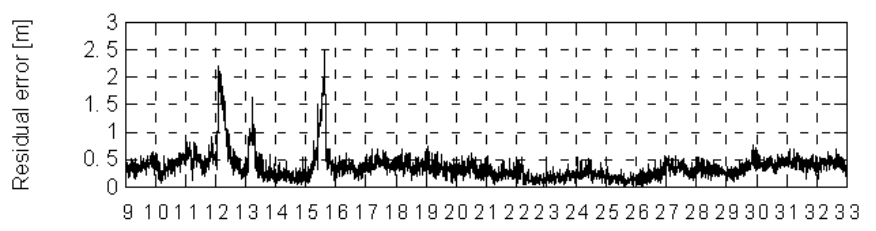

(d)

Local time [hour]

Fig. 3. (a) Comparison of estimated vertical TEC from single and dual frequency measurement, (b) Longitudinal and latitudinal TEC gradients, (c) comparison of estimated receiver clock error from single and dual frequency measurement, (d) estimation error of receiver clock error

The TEC errors estimated by the proposed method is discussed. Figure. 4 shows the histogram of the estimated TEC errors to those derived from the dual frequency measurements, that is differences between red and black lines in figure 3 (a). The horizontal axis shows the TEC errors in TECU and vertical axis does counts. The average of the TEC error is 0.87 TECU and the standard deviation is 8.52 TECU. There is no artificial bias of the estimated TEC.

The proposed method was applied to data obtained at other 680 GEONET stations and examined their TEC errors. Figure 5 shows spatial distribution of average of the TEC errors. In the figure, the average less than -3.16 TECU are shown by red pluses, between -3.16 TECU and 0 are by green crosses, between 0 and +3.16 TECU are as blue stars, and larger than $+3.16 \mathrm{TECU}$ are as magenta rectangles. From the figure, a clear regional dependence of the average of TEC errors is found. As the location moves to south, large bias errors appear. In the east side, larger negative bias regions are also found, as shown by the red pluses.

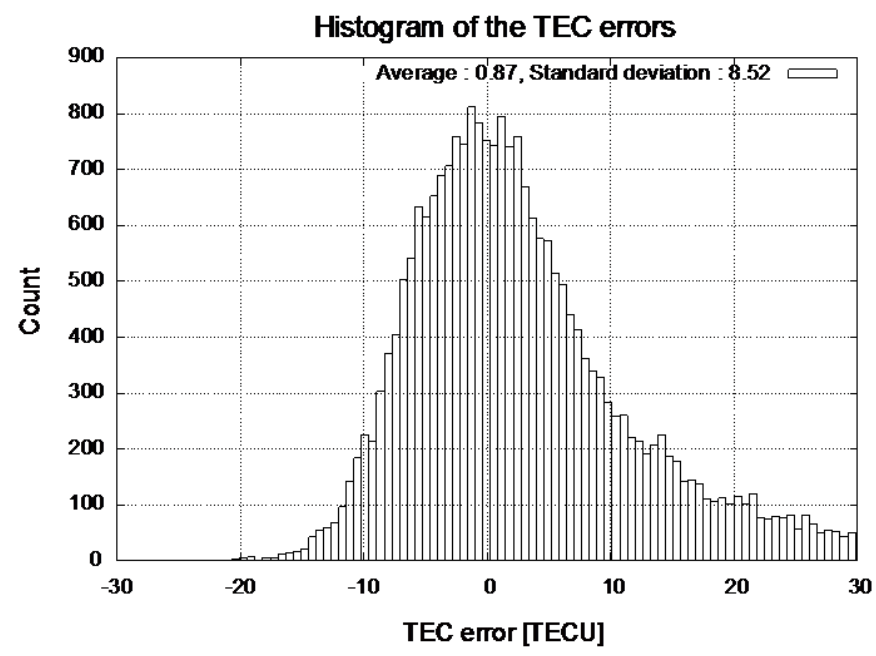

Fig. 4. Histogram of the TEC errors of the proposed method applied on Uchinada data on 2013 Nov. 10

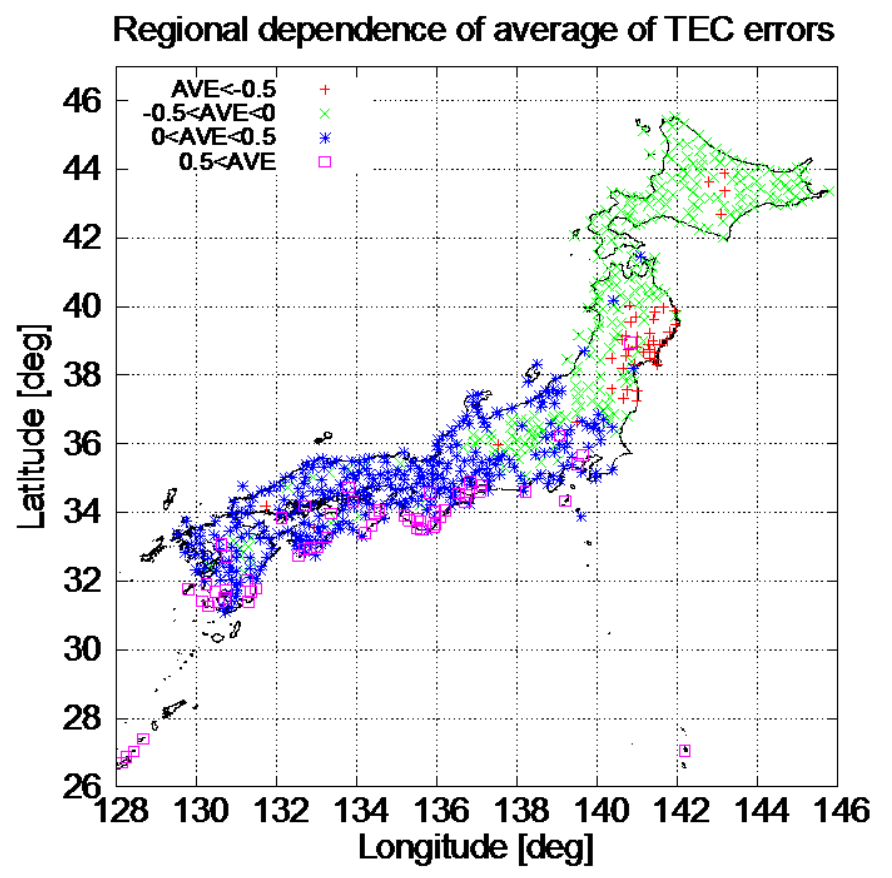

Fig. 5. Spatial distribution of average of the TEC errors

The regional dependences of the standard deviation of the TEC errors were also examined. The result is shown in Figure 6. In the figure, the standard deviation less than 9.2 are shown by red pluses, between 9.2 and 12.3 are by green crosses, between 12.3 and 18.5 are by blue stars, and larger than 18.5 are by magenta rectangles. The largest standard deviation is found at around $\left(142^{\circ} \mathrm{E}, 38^{\circ} \mathrm{N}\right)$ and it becomes smaller as the location moves away from there.

From the regional dependences of the average and standard deviation of the estimated TECs, it is found that the accuracy of the proposed method deeply depends on the 
assumption of the spatial distribution of the TEC. In the method, the TEC variation is assumed to be represented by $1^{\text {st }}$ order gradient for both latitude and longitude. From the average distribution, however, when the receiver is located at lower latitudes where a large latitudinal TEC gradient exists in a typical daytime, accuracy of the TEC estimation becomes worse. This result implies that the latitudinal distribution cannot be represented by $1^{\text {st }}$ order formula in such regions. To apply the method to data obtained at low latitude regions, higher order function should be required.

Another restriction of the method is found in the standard deviation map. There is no regional dependence of performance of the receivers in GEONET, and thus the large standard deviations found at $\left(142^{\circ} \mathrm{E}, 38^{\circ} \mathrm{N}\right)$ is due to other effects. In the terms in equation (1), only $\delta_{\text {ion }}^{i}$ and $\delta_{\text {tro }}^{i}$ have regional dependences. Since the tropospheric delay $\delta_{\text {tro }}^{i}$ is much smaller than the ionospheric delay $\delta_{\text {ion }}^{i}$, the large standard deviation is considered to be caused by complex TEC distributions which cannot be represented by the $1^{\text {st }}$ order model. It should be noted that the proposed method cannot adapt such complex TEC structures.

\section{Regional dependence of standard deviation of TEC errors}

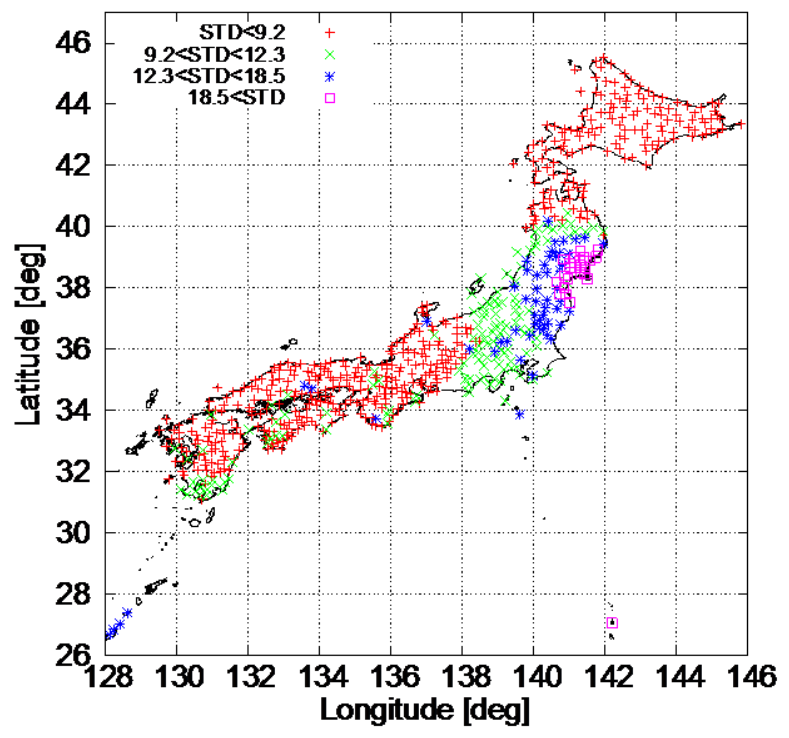

Fig. 6. Spatial distribution of standard deviation of the TEC errors

\section{CONCLUSION}

In this paper, an estimation method of TEC distribution map from GPS signals was proposed. A remarkable point of the method is that it only uses single frequency measurements while the TEC observation has been conducted by dual frequency measurements. By this method, it becomes easy to construct TEC observation networks at low costs where the GPS networks are not yet installed.

In the developed method, the TEC distributions is assumed to be represented by a $1^{\text {st }}$ order gradient model for latitude and longitude. This assumption sometimes causes large TEC errors when the TEC distributions have unexpected structures. It was confirmed that TEC gradients from low to mid latitudes in a daytime cannot be represented by the model. To solve this problem, higher order formula, such as $2^{\text {nd }}$ order polynomial function, is available. As higher order functions are adopted, the number of parameters to be solved increases. By using data from multiple receivers, several independent equations can be used while its receiver clock error should be estimated as an additional parameter. This modification remains to be solved in the future study. We are planning to build observation networks of ionospheric TEC distribution at lower latitude regions, where the networks are not yet installed, at low cost.

\section{ACKNOWLEGEMENTS}

The code pseudorange data used in this study are provided by Geospatial Information Authority of Japan. The ionospheric vertical TEC map and the orbital data of the GPS satellites are provided by the International GNSS Service (IGS).

\section{REFERENCES}

[1] T. Ondoh and K. Marubashi eds., Science of Space Environment, Ohmsha, IOS Press, 2001.

[2] M. Hernández-Pajares, J. M. Juan, J. Sanz, R. Orus, A. Garcia-Rigo, J. A. Feltens, S. C. Komjathy, Schaer \& Krankowski, A. (2009). The IGS VTEC maps: a reliable source of ionospheric information since 1998. Journal of Geodesy, Vol. 83 (3-4), pp. 263-275.

[3] Feltens, J. Dow, J.M. Realized and planned improvements in ESA/ESOC ionosphere modelling, IGS Workshop 2006.

[4] Zishen Li, Yunbin Yuan, Ningbo Wang, Manuel Hernandez-Pajares, Xingliang Huo (2015). SHPTS: towards a new method for generating precise global ionospheric TEC map based on spherical harmonic and generalized trigonometric series functions. Journal of Geodesy.

[5] H. P. Zhang, W. Xu, M. Han, Ge and C. Shi, Eliminating negative VTEC in global ionosphere maps using inequality-constrained least squares, Advances in Space Research Vol. 51, No. 6, 2013, pp. 9881000.

[6] J. M. Dow, R. E. Neilan, and C. Rizos, The international GNSS service in a changing landscape of global navigation satellite systems, Journal of Geodesy, 83:191-198, DOI: 10.1007/s00190-008-0300-3, 2009.

[7] International GNSS Service (IGS). Ionosphere Product. NASA CDDIS. Available from <ftp://cddis.gsfc.nasa.gov/gnss/products/ionex/>

[8] S. Schaer, W. Gurtner, and J. Feltens, IONEX: The IONosphere Map Exchange Format Version 1, Proc. Of the IGS AC Workshop.

[9] J. G. Grimes, GPS Standard Positioning Service (SPS) Performance Standard 4th Ed, 2008.

[10] M. J. Dunn, Global Positioning Systems Directorate Systems Engineering and Integration, Interface Specification, IS-GPS-200H, 2013.

[11] G. Xu, GPS Theory, Algorithms and Applications 2nd Ed, Springer, 2007.

[12] P. Misra, P. Enge, Global Positioning System, Signals, Measurements, and Performance, Revised Second Edition, 2011.

[13] J. A. Klobuchar, Ionospheric time-delay algorithm for single-frequency GPS users, IEEE Trans. on Aero. and Elec. Sys., AES-23, 3, 1987. 